\title{
In contrast to melanocytes, keratinocytes of normal human adult interfollicular epidermis do not express galectin-1
}

\author{
Efstathia Pasmatzi ${ }^{1}$, Christina Papadionysiou ${ }^{1}$, George Badavanis², Nikiforos Kapranos ${ }^{3}$, Alexandra Monastirli, ${ }^{1,2}$, \\ Dionysios Tsambaos ${ }^{1,2}$
}

${ }^{1}$ Department of Dermatology, School of Medicine, University of Patras, Patras, Greece. ${ }^{2}$ Center for Dermatologic Diseases, Limassol, Cyprus. ${ }^{3}$ Laboratory for Molecular Histopathology, Athens, Greece.

\begin{abstract}
Introduction: Galectins constitute a phylogenetically conserved family of proteins that specifically bind to glycoconjugates bearing $\beta$-galactoside residues. Although galectin-1 ( $\mathrm{Gal}$ 1), the first identified member of the galectin family, is involved in highly important biological processes at the molecular and cellular level in human skin, its expression in keratinocytes of normal human adult interfollicular epidermis (NHAIE) remains in dispute, whereas that in epidermal melanocytes has drawn very little attention so far. This prompted us to investigate the expression of Gal 1 in the keratinocytes and melanocytes of NHAIE.

Methods: Biopsy specimens obtained from the buttock skin of 23 healthy adult volunteers of both sexes were processed for single and double immunohistochemical staining using antibodies against Gal 1 and Melan-A.

Results: In contrast to epidermal melanocytes, which revealed a distinct Gal 1 immunoreactivity, keratinocytes of NHAIE were completely devoid of any expression of this galectin.

Conclusions: This article simultaneously assesses Gal 1 immunoreactivity of keratinocytes and melanocytes in NHAIE for the first time. Our findings may contribute to a better understanding of alterations in Gal 1 expression in various benign and malignant cutaneous disorders and may be of importance for the future design of targeted therapies.
\end{abstract}

Keywords: galectin-1, melanocytes, epidermal keratinocytes, human epidermis

Received: 24 December 2019 | Returned for modification: 23 March 2020| Accepted: 24 March 2020

\section{Introduction}

Galectins constitute a phylogenetically conserved family of proteins that are expressed in a wide range of species and demonstrate a high binding affinity for $\beta$-galactose-rich glycoconjugates. Most galectins share a unique structural unit termed the carbohydrate-recognition domain (CRD), which is made up of about 130 amino acids. Some galectins, however, possess two homologous CRDs in a single polypeptide chain separated by a linker of up to 70 amino acids (1-3).

Thirteen out of the 19 galectins identified so far show a widespread occurrence in human tissues, but only a small number of them are characterized by high tissue specificity. Subsequent to their synthesis in the cytosolic ribosomes, galectins can be transferred to the nucleus or other subcellular sites and exported from cells through non-classical secretion, thus occurring at the cell surface and extracellularly as well $(4,5)$. Recent accumulating evidence suggests that galectins are involved in a diverse array of important biological processes at the molecular and cellular levels in the skin and other tissues (6).

Galectin-1 (Gal 1), the first-identified and best-studied of the galectin family, is encoded in humans by the LGALS1 gene, which is located on chromosome 22 (q12) (7). Gal 1 is a noncovalent homodimeric protein with a $14 \mathrm{kDa}$ monomer, which contains one CRD and preferentially recognizes galactose- $\beta 1-4-\mathrm{N}$-acetyl-glucosamine sequences on $\mathrm{N}$ - or O-linked glycans. Gal 1 occurs intracellularly (in the cytoplasm and the nucleus) and remains there until cell activation $(8,9)$. Gal-1 secretion to the cell surface and into the extracellular matrix (ECM) occurs to a lesser extent. This galectin is dif- ferentially expressed in various normal and pathological tissues and can act at both the intracellular level (as an effector of premRNA splicing) and the extracellular level as a binding protein for diverse glycoconjugates and constituent elements of ECM (10).

Although the multifaceted biological properties and actions of Gal 1 (Table 1) are involved in highly important processes at the molecular and cellular levels in epithelial and nonepithelial tissues (11-13), the immunoreactivity for this galectin in keratinocytes of normal human adult interfollicular epidermis (NHAIE) still remains in dispute, whereas that of epidermal melanocytes has drawn very little attention so far. This prompted us to investigate the expression of Gal 1 in keratinocytes and melanocytes of NHAIE.

Table 1 | The most significant biological processes in the skin and extracutaneous tissues in which Gal 1 is involved.

\section{Process}

Morphogenesis

Apoptosis

Angiogenesis

Differentiation of hematopoietic and myogenic lineage

Regulation of cell cycle and proliferation

T-cell homeostasis and survival

Regulation of the innate and the adaptive immune response

Cell-cell and cell-matrix adhesion

Inflammation

Cell motility

Tumor angiogenesis, hypoxia, invasion, and metastasis

Autoimmune and allergen-induced inflammation

Induction of B cells' regulatory function

Escape of tumor cells from immune surveillance

Microglial modulation, polarization, and remyelination 


\section{Material and methods}

Subsequent to the approval of the study protocol by the local ethics committee, biopsy specimens obtained from the buttock skin of 23 healthy adult volunteers of both sexes (23-31 years old), who provided written consent, were processed for single and double immunohistochemistry.

The expression of Gal 1 and Melan-A was investigated by single immunohistochemistry in $4 \mu \mathrm{m}$ sections of formalin-fixed and paraffin-embedded skin specimens with the peroxidase-labeled streptavidin-biotin standard immunohistochemical technique (14) using commercially available monoclonal antibodies against Melan-A (Dako, Clone A103), Gal 1 (Novocastra, Clone, 25C1, concentration 1:100), and 3,3'-diaminobenzidine solution (Sigma, Munich, Germany) as a chromogen.

The same antibodies were also used for double immunochemistry (15). Sections were consecutively incubated with Dako Envision FLEX/HRP solution and finally 3,3'-diaminobenzidine $\mathrm{H}_{2} \mathrm{O}_{2}$ containing solution (Sigma, Munich, Germany) for the detection of Melan-A (Dako, Clone A103) and with HRP Magenda DAKO solution containing an alternative red chromogen, 3-amino-9-ethylcarbazole (AEC), for the detection of Gal 1 antibody (Novocastra, Clone, $25 \mathrm{C} 1$, concentration 1:100).

\section{Results}

In all specimens of NHAIE examined the immunohistochemical results were identical, and thus they are described here together. Keratinocytes of all epidermal layers were completely devoid of any Gal 1 immunoreactivity, whereas epidermal melanocytes, mostly found in the basal layer of the epidermis, revealed strong expression of Gal 1 (Fig. 1A). The basement membrane revealed no Gal 1 immunoreactivity, whereas spindle mesenchymal cells and endothelial cells of the capillaries in the papillary dermis exhibited a strong expression of this galectin. Epidermal melanocytes demonstrated a strong immunoreactivity for Melan-A (Fig. 1B). On double immunohistochemistry, epidermal melanocytes showed strong immunoreactivity for both Melan-A and Gal 1 (Fig. 2), whereas keratinocytes were completely negative.

\section{Discussion}

Surprisingly, the expression of Gal 1 in the melanocytes of NHAIE has received very little scientific attention over the years. To the best of our knowledge, this issue has only been studied by Bolander et al. (7), who used tissue microarrays and immunohistochemistry with the melanocyte marker Melan-A and found strong expression of Gal 1 in normal epidermal melanocytes. In this study, using double immunostaining (Gal 1 and Melan-A), we also detected strong Gal 1 immunoreactivity in the melanocytes of NHAIE, thus confirming the findings of Bolander et al. (7).

The results of recent studies have shown that Gal 1 is overexpressed in melanomas (particularly in aggressive ones), and that this galectin is also involved in the mechanisms underlying the escape of melanoma cells from immune surveillance, metastatic progression, and the protection of melanomas from the cytotoxic effects of chemotherapy and radiotherapy $(13,16-18)$. These findings, together with the immunosuppressive, proangiogenic, and tumorigenic potential of Gal 1, support the hypothesis that this galectin may serve as a promising molecular target for the development of new Gal 1 inhibitors that could be used either alone or

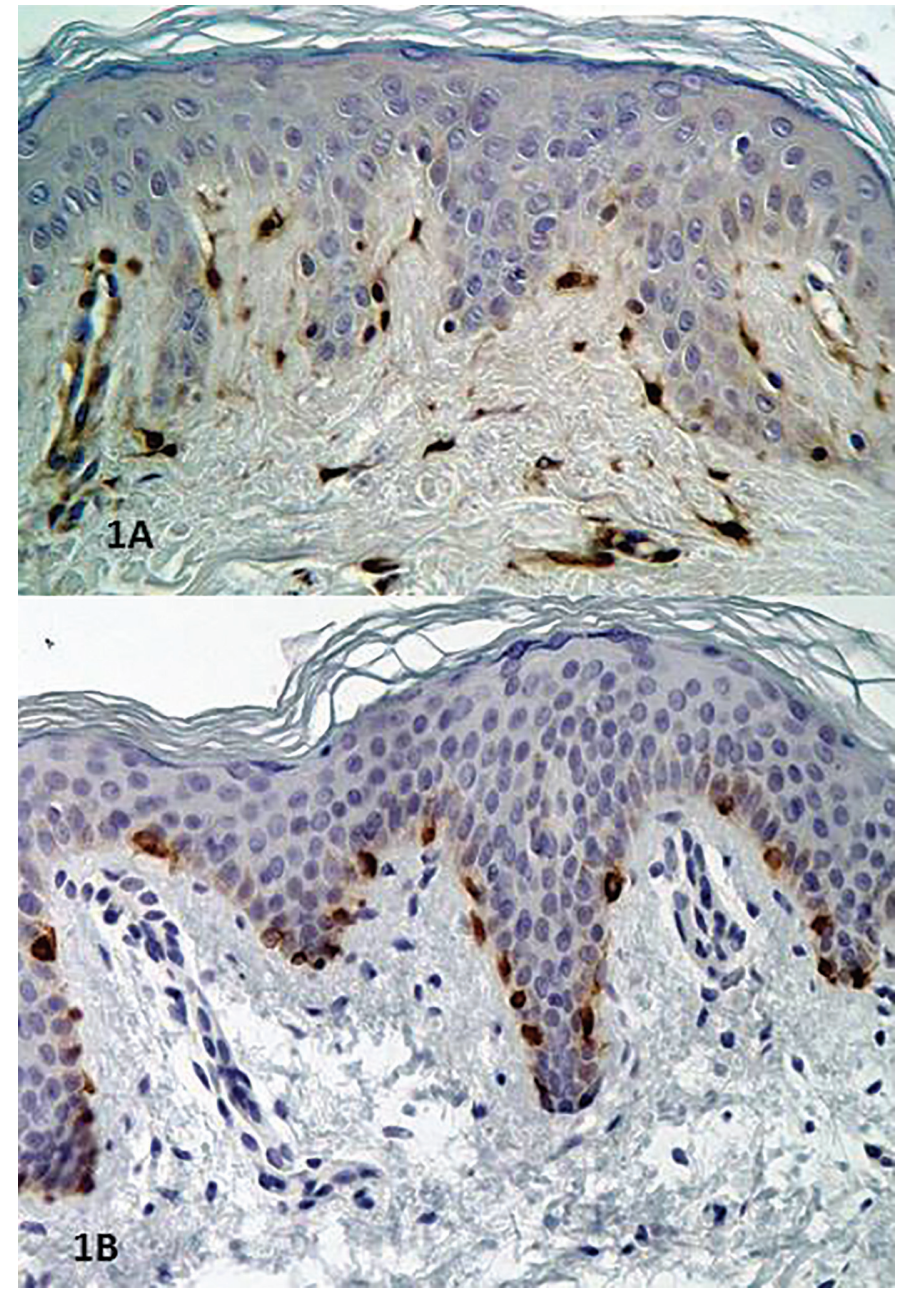

Figure $1 \mid$ A) Keratinocytes in all cell layers of normal human adult interfollicular epidermis (NHAIE) are devoid of any Gal 1 immunoreactivity, whereas there is a distinct expression of this galectin in the epidermal melanocytes of the basal layer, in the endothelial cells of capillaries, and in the mesenchymal cells of the papillary dermis (original magnification $\times 40$ ). B) Positive Melan-A immunoreactivity of melanocytes in NHAIE (original magnification $\times 40$ ).

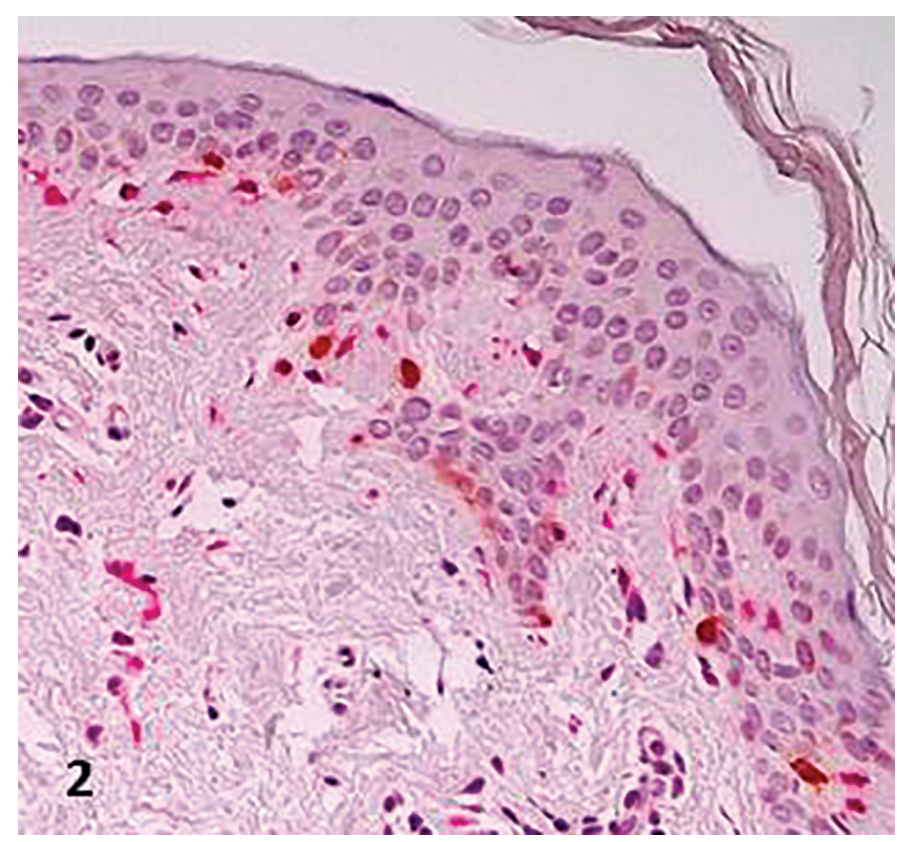

Figure 2 | On double immunohistochemistry, positive immunoreactivity of melanocytes of normal human adult interfollicular epidermis (NHAIE) to both Gal 1 (red) and Melan-A (brown) is observed (original magnification $\times 20$ ).

in combination with chemotherapy and radiotherapy in the fight against melanoma (19-21). 
The results of previous studies on the expression of Gal 1 in the keratinocytes of NHAIE are contradictory (Table 2). Thus, Gal 1 immunoreactivity reportedly occurs in the basal and suprabasal keratinocytes of NHAIE (22-25) and also in the corneal keratinocytes (26). However, other research groups have detected no expression of Gal 1 in the keratinocytes of all layers of $\operatorname{NHAIE}(27,28)$. The explanation of the above conflicting results is very difficult; nevertheless, it is possible that these results may be at least partially due to differences in the methodological procedures applied.

Table 2 | Conflicting results with regard to Gal 1 expression in keratinocytes and melanocytes of normal human adult interfollicular epidermis.

\begin{tabular}{|c|c|c|}
\hline Authors & Keratinocytes & Melanocytes \\
\hline Allen et al. (1991) & Positive* (BC, SBC) & ND \\
\hline Akimoto et al. (1995) & Positive (BC, SBC) & ND \\
\hline Holíková et al. (2002) & Positive (BC, SBC) & ND \\
\hline Wada et al. (2003) & Positive (BC, SBC) & ND \\
\hline Klíma et al. (2005) & Positive (BC, SBC, COR) & ND \\
\hline Lacina et al. (2006) & Negative (BC, SBC, COR) & ND \\
\hline Bolander et al. (2008) & ND & Positive \\
\hline Cada et al. (2009) & Negative (BC, SBC, COR) & ND \\
\hline This study & Negative (BC, SBC, COR) & Positive \\
\hline
\end{tabular}

This study was unable to detect any Gal 1 immunoreactivity in the keratinocytes of all layers on NHAIE, thus confirming the findings of Lacina et al. (26) and Cada et al. (28). In addition, in other immunohistochemical studies performed in our lab on the apparently normal skin of adult patients with diverse benign disorders (lichen planus, psoriasis, and prurigo nodularis), all epidermal keratinocytes of the interfollicular epidermis were found to be completely devoid of any Gal 1 immunoreactivity, irrespective of the degree of their differentiation, whereas there was distinct Gal 1 expression in keratinocytes of the hair follicles (unpublished data).

Interestingly, keratinocytes of normal human oral mucosa also reveal no Gal 1 expression (29), whereas head, neck, and oral cavity squamous cell carcinomas (SCCs) demonstrate overexpression of this galectin (30). In particular, in head and neck SCCs, upregulation of Gal 1 expression significantly correlates with the presence of cancer-associated stromal myofibroblasts and the activation of genes related to known poor-prognosis factors for SCCs (31). Moreover, in view of the high sensitivity of Gal 1 immunoreactivity in the detection of neoplastic cells in oral and head and neck SCCs, it has been suggested that this galectin may represent a useful immunocytochemical and prognostic marker for SCCs, which is strongly associated with the malignant transformation of keratinocytes $(32,33)$. Thus, targeting of Gal 1 by non-peptidic inhibitors may represent a promising approach in the treatment of patients with SCCs (33).

\section{Conclusions}

Gal 1 immunoreactivity in keratinocytes and melanocytes in NHAIE is simultaneously assessed for the first time in this article and the results reveal that, in contrast to melanocytes, keratinocytes in NHAIE are completely devoid of any Gal 1 immunoreactivity. These findings may contribute to a better understanding of alterations in Gal 1 expression in various benign and malignant cutaneous disorders and may be of importance for the future design of targeted therapies.

\section{References}

1. Larsen L, Chen HH, Saegusa J, Liu FT. Galectin-3 and the skin. J Derm Sci. 2011; 64:85-91.

2. Kobayashi JN. Tissue- and cell-specific localization of galectins, b-galactosebinding animal lectins, and their potential functions in health and disease. Anat Sci Int. 2017;92:25-36.

3. Pasmatzi E, Papadionysiou C, Badavanis G, Monastirli A, Tsambaos D. Galectin 3: an extraordinary multifunctional protein in dermatology: current knowledge and perspectives. Anal Bras Dermatol. 2019;94:348-54.

4. Liu FT, Bevins CL. A sweet target for innate immunity. Nat Med. 2010;16:263-4.

5. Dings RPM, Miller MC, Griffin RJ, Mayo KH. Galectins as molecular targets for therapeutic intervention. Int J Mol Sci. 2018;19:905.

6. Tian Y, Yuan W, Li J, Wang H, Hunt MG, Liu C, et al. TGF $\beta$ regulates galectin-3 expression through canonical Smad3 signaling pathway in nucleus pulposus cells: implications in intervertebral disc degeneration. Matrix Biol. 2016;50:39-52.

7. Bolander A, Agnarsdottir M, Stromberg S, Ponten F, Hesselius P, Uhlen M, et al. The protein expression of TRP-1 and galectin-1 in cutaneous malignant melanomas. Cancer Genomics \& Proteom. 2008;5:293-300.

8. Cho M, Cummings RD. Galectin-1, a $\beta$-galactoside-binding lectin in Chinese hamster ovary cells: II. Localization and biosynthesis. J Biol Chem. 1995;270:520712.

9. Shalom-Feuerstein R, Cooks T, Raz A, Kloog Y. Galectin-3 regulates a molecular switch from N-Ras to K-Ras usage in human breast carcinoma cells. Cancer Res. 2005;65:7292-300.

10. Verschuere T, van Woensel M, Fieuws S, Lefranc F, Mathieu V, Kiss R, et al. Altered galectin-1 serum levels in patients diagnosed with high-grade glioma. J Neurooncol. 2013;115:9-17.

11. Elola MT, Chiesa ME, Alberti AF, Mordoh J, Fink NE. Galectin-1 receptors in different cell types. J Biomed Sci. 2005;12:13-29.

12. Camby I, Le Mercier M, Lefranc F, Kiss R. Galectin-1: a small protein with major functions. Glycobiology 2006; p. 137R-157R.

13. Pasmatzi E, Papadionysiou C, Badavanis G, Monastirli A, Tsambaos D. Galectin 1 in dermatology: current knowledge and perspectives. Acta Dermatovenereol Alp Pannonica Adriat. 2019;28:27-31.

14. Leonardi R, Villari L, Caltabiano M, Travali S. Heat shock protein 27 expression in the epithelium of periapical lesions. J Endod. 2001;27:89-92.
15. Chen X, Cho DB, Yang PC. Double staining immunohistochemistry. N Am J Med Sci. 2010;2:241-5.

16. Rubinstein N, Alvarez M, Zwirner NW, Toscano MA, Ilarregui JM, Bravo A, et al. Targeted inhibition of galectin-1 gene expression in tumor cells results in heightened T cell-mediated rejection; a potential mechanism of tumor-immune privilege. Cancer Cell. 2004;5:241-51.

17. Lefranc F, Mathieu V, Kiss R. Galectin-1 as an oncotarget in gliomas and melanomas. Oncotarget. 2011;2:892-3.

18. Mathieu V, de Lassalle EM, Toelen J, Mohr T, Bellahcène A, Van Goietsenoven G, et al. Galectin-1 in melanoma biology and related neo-angiogenesis processes. J Invest Dermatol. 2012;132:2245-54.

19. Astorgues-Xerri L, Riveiro ME, Tijeras-Raballand A, Serova M, Neuzillet C, Albert $S$, et al. Unraveling galectin-1 as a novel therapeutic target for cancer. Cancer Treat Rev. 2014;40:307-19.

20. Wdowiak K, Francuz T, Gallego-Colon E, Ruiz-Agamez N, Kubeczko M, Grochoła I, et al. Galectin targeted therapy in oncology: current knowledge and perspectives. Int J Mol Sci. 2018;19:210.

21. Goud NS, Soukya PSL, Ghouse M, Komal D, Alvala R, Alvala M. Human galectin-1 and its inhibitors: privileged target for cancer and HIV. Mini Rev Med Chem. 2019;19:1369-78.

22. Allen HJ, Gottstine S, Sharma A, DiCioccio RA, Swank RT, Li H. Synthesis, isolation, and characterization of endogenous beta-galactoside-binding lectins in human leukocytes. Biochem. 1991;30:8904-10.

23. Akimoto Y, Hirabayashi J, Kasai K, Hirano H. Expression of the endogenous $14^{-}$ $\mathrm{kDa}$ beta-galactoside-binding lectin galectin in normal human skin. Cell Tissue Res. 1995;280:1-10.

24. Holíková Z, Hrdlicková-Cela E, Plzák J, Smetana Jr K, Betka J, Dvoránková B, et al. Defining the glycophenotype of squamous epithelia using plant and mammalian lectins. Differentiation-dependent expression of alpha2,6- and alpha2,3linked $\mathrm{N}$-acetylneuraminic acid in squamous epithelia and carcinomas and its differential effect on binding of the endogenous lectins galectins-1 and -3. APMIS. 2002;110:845-56.

25. Wada M, Ono S, Kadoya T, Kawanami T, Kurita K, Kato T. Decreased galectin-1 immunoreactivity of the skin in amyotrophic lateral sclerosis. J Neurol Sci. 2003; 208:67-70. 
26. Klíma J, Smetana Jr K, Motlík J, Plzáková Z, Liu F-T, Stork J, et al. Comparative phenotypic characterization of keratinocytes originating from hair follicles. J Mol Histol. 2005;36:89-96.

27. Lacina L, Plzáková Z, Smetana Jr K, Stork J, Kaltner H, André S. Glycophenotype of psoriatic skin. Folia Biol (Praha). 2006;52:10-5.

28. Cada Z, Smetana Jr K, Lacina L, Plzáková Z, Stork J, Kaltner H, et al. Immunohistochemical fingerprinting of the network of seven adhesion/growth-regulatory lectins in human skin and detection of distinct tumour-associated alterations. Folia Biol (Praha). 2009;55:145-52.

29. Noda Y, Kishino M, Sato S, Hirose K, Sakai M, Fukuda Y, et al. Galectin-1 expression is associated with tumour immunity and prognosis in gingival squamous cell carcinoma. J Clin Pathol. 2017;70:126-33.

30. Chiang WF, Liu SY, Fang LY, Lin CN, Wu MH, Chen YC, et al. Overexpression of galectin-1 at the tumor invasion front is associated with poor prognosis in earlystage oral squamous cell carcinoma. Oral Oncol. 2008;44:325-34.
31. Valach J, Fík Z, Strnad H, Chovanec M, Plzák J, Cada Z, et al. Smooth muscle ac tin-expressing stromal fibroblasts in head and neck squamous cell carcinoma: increased expression of galectin-1 and induction of poor prognosis factors. Int Cancer. 2012;131:2499-508.

32. Noda Y, Kondo Y, Sakai M, Sato S, Kishino M. Galectin-1 is a useful marker for detecting neoplastic squamous cells in oral cytology smears. Hum Pathol. 2016; 52:101-9.

33. Koonce NA, Griffin RJ, Dings RPM. Galectin-1 inhibitor OTXoo8 induces tumor vessel normalization and tumor growth inhibition in human head and neck squamous cell carcinoma models. Int J Mol Sci. 2017;9:18. 